\title{
Genomic and transcriptomic comparison of post-radiation versus sporadic sarcomas
}

\author{
Tom Lesluyes $\mathbb{1}^{1,2,3,4} \cdot$ Jessica Baud ${ }^{1,2} \cdot$ Gaëlle Pérot ${ }^{1,5} \cdot$ Céline Charon-Barra $^{6} \cdot$ Axel You $^{1,7} \cdot$ Isabelle Valo ${ }^{8}$. \\ Céline Bazille $^{9}$. Florence Mishellany ${ }^{10}$ - Agnès Leroux ${ }^{11}$. Sophie Renard-Oldrini ${ }^{12}$. Philippe Terrier $\mathbb{D}^{13}$. \\ Axel Le Cesne ${ }^{14}$ - Marick Laé ${ }^{15,16}$. Sophie Piperno-Neumann ${ }^{17}$. Sylvie Bonvalot $\mathbb{C}^{18}$ - Agnès Neuville ${ }^{5,20}$.

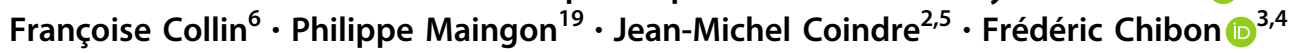

Received: 28 March 2019 / Revised: 7 May 2019 / Accepted: 8 May 2019 / Published online: 26 June 2019

(c) United States \& Canadian Academy of Pathology 2019

\begin{abstract}
Post-radiation sarcomas are rare secondary cancers arising from radiation therapies. To date, few genetic specificities have been described for such malignancies and the oncogenesis of sarcomas with complex genetics (both sporadic and postradiation) remains largely misunderstood. We performed genomic and transcriptomic analyses on 77 post-radiation sarcomas using DNA-array and RNA sequencing. Consequently, we were able to investigate changes in copy number variations, transcriptome profiling, fusion gene expression, and mutational landscapes. We compare these data to a reference cohort of 93 sporadic sarcomas. At genomic level, similar chromosomal complexity was observed both in post-radiation and sporadic sarcomas with complex genetics. We found more frequent $C D K N 2 A$ and $C D K N 2 B$ (coding for $\mathrm{p} 14 / \mathrm{p} 16$ and p15 proteins, respectively; at $9 \mathrm{p} 21.3)$ losses in post-radiation $(71 \%)$ than in sporadic tumors $(39 \% ; P=6.92 \mathrm{e}-3)$. Among all detected fusion genes and punctual variations, few specificities were observed between these groups and such alterations are not able to drive a strong and specific oncogenesis. Recurrent $M Y C$ amplifications (96\%) and KDR variants (8\%) were detected in post-radiation angiosarcomas, in agreement with the literature. Transcriptomic analysis of such angiosarcomas revealed two distinct groups harboring different genomic imbalances (in particular gains of 17q24.2-17qter) with different clinical courses according to patient's vital status. Differential gene expression analysis permitted to focus on the immune response as a potential actor to tumor aggressiveness. Histochemistry validated a lower inflammation and lower immune infiltrate at tumor periphery for highly aggressive angiosarcomas. Our results provide new genomic and transcriptomic information about postradiation sarcomas. The techniques we used (RNA-seq and DNA-arrays) did not highlight major differences in sarcomas with complex genetics depending on the radiation context, revealing similar patterns of transcriptomic profiles and chromosomal copy number variations. Additional characterizations, particularly whole genome sequencing, could measure changes in DNA following radiation therapy in such malignancies and may precise their oncogenesis.
\end{abstract}

\section{Introduction}

Post-radiation sarcomas (also known as secondary sarcomas or radiation-induced sarcomas) represent a small fraction of all sarcomas $(<5 \%)$ and such second malignancies arise from previous radiation therapies [1-3]. Firstly established

Supplementary information The online version of this article (https:// doi.org/10.1038/s41379-019-0300-2) contains supplementary material, which is available to authorized users.

Frédéric Chibon

frederic.chibon@inserm.fr

Extended author information available on the last page of the article in 1948 [4] and reviewed in 1971 [5], post-radiation sarcomas are defined by three criteria: a latency period of at least 3 years prior to the newly diagnosed sarcoma, sarcoma located in the radiation area of the previous cancer, and a histological difference between the primary tumor and the sarcoma. Though this secondary tumor is generally diagnosed after a decade, a shorter period (6 months) has also been proposed if the other criteria are fulfilled [6].

Despite the incontestable benefits of radiation therapy on cancer, a small fraction of patients develops a secondary tumor $(<1 \%)$ [7]. Survival studies show that post-radiation sarcomas are more aggressive than sporadic ones [6, 8-11]. This can be explained by several factors: unfavorable tumor locations, poor treatment responses, late diagnoses, elderly 
patients, necrosis, and frequent metastatic outcomes [12, 13]. In addition, these groups have distinct clinical characteristics. For example, undifferentiated pleomorphic sarcomas, osteosarcomas, and angiosarcomas have higher incidences as postradiation compared to sporadic tumors [9-11, 13, 14].

Ionizing radiation is known to alter DNA [15]. It creates genomic alterations and disables DNA-repair mechanisms. Post-radiation sarcomas predominantly develop in zones exposed to high doses (50 Gy, normally inducing cell death) rather than low doses ( $30 \mathrm{~Gy}$, generating genomic instability) $[8,9]$. On the other hand, carcinomas are observed in areas exposed to low doses, even though a link between radiation dose and cumulative incidence rate has been suggested [16].

To date, few genetic differences have been observed between sporadic and post-radiation tumors. For example, post-radiation angiosarcomas harbor recurrent $M Y C$ amplifications ( $>50 \%$; a well-known proto-oncogene) [17] and $K D R$ mutations (10\%; coding for the vascular endothelial growth factor receptor 2) [18], unlike sporadic angiosarcomas. This suggests that the oncogenic mechanism may be different in these two models. However, other post-radiation subtypes do not harbor specific genomic alterations, which could be related to different biological pathways from sporadic sarcomas, whose oncogenesis remains unclear. Post-radiation sarcomas with complex genetics have similar genomic alterations to sporadic ones, such as $R B 1$ loss and $\mathrm{p} 53$ pathway inactivation [19-21]. Nevertheless, a sarcoma-based gene expression signature, representing chronic oxidative stress, has been proposed to distinguish the two entities [22]. In addition, several gene-sets have been proposed as indicators of irradiation in various human models [23-28].

We consequently studied the genome and transcriptome characteristics of post-radiation sarcomas to better understand the biology of these tumors. We used DNA-arrays to measure their genomic alterations and performed RNA sequencing (RNA-seq) to explore their transcriptome profiles.

\section{Material and methods}

\section{Cohorts}

To highlight differences between post-radiation and sporadic sarcomas, we compared our results to an independent cohort of sporadic tumors. Clinical characteristics are available in Table 1. Additional information about patient recruitment, histological review, molecular profiling, and data processing/ filtering can be found in the Supplementary Methods.

\section{Data accession}

The 93 sporadic sarcomas are part of cohort \#2 (95 cases) previously published [29], except cases N001 and S915 for
Table 1 Clinical characteristics in the two independent cohorts: sporadic and post-radiation sarcomas

\begin{tabular}{|c|c|c|}
\hline Cohort & $\begin{array}{l}93 \text { sporadic } \\
\text { sarcomas }\end{array}$ & $\begin{array}{l}77 \text { post- } \\
\text { radiation } \\
\text { sarcomas }\end{array}$ \\
\hline Males & $53(56.99 \%)$ & $14(18.18 \%)$ \\
\hline Females & $40(43.01 \%)$ & $63(81.82 \%)$ \\
\hline Age at diagnosis & $65[62-68]$ & $67[63-72]$ \\
\hline Tumor size (mm) & $100[90-120]$ & $50[45-60]$ \\
\hline \multicolumn{3}{|l|}{ Tumor site } \\
\hline Gynecological area & 0 & $1(1.30 \%)$ \\
\hline Head and neck & 0 & $14(18.18 \%)$ \\
\hline Internal trunk & $18(19.35 \%)$ & 0 \\
\hline Extremity & $58(62.37 \%)$ & $7(9.09 \%)$ \\
\hline Trunk wall & $17(18.28 \%)$ & $55(71.43 \%)$ \\
\hline \multicolumn{3}{|l|}{ FNCLCC grade } \\
\hline 1 & $3(3.23 \%)$ & $4(5.19 \%)$ \\
\hline 2 & $22(23.66 \%)$ & $18(23.38 \%)$ \\
\hline 3 & $64(68.82 \%)$ & $46(59.74 \%)$ \\
\hline Unknown & $4(4.30 \%)$ & $9(11.69 \%)$ \\
\hline \multicolumn{3}{|l|}{ Histological subtype } \\
\hline Angiosarcoma & 0 & $24(31.17 \%)$ \\
\hline Dedifferentiated liposarcoma & $13(13.98 \%)$ & $3(3.9 \%)$ \\
\hline Leiomyosarcoma & $22(23.66 \%)$ & $11(14.29 \%)$ \\
\hline Myxofibrosarcoma & $12(12.90 \%)$ & $6(7.79 \%)$ \\
\hline Others & $4(4.30 \%)$ & $12(15.58 \%)$ \\
\hline Pleomorphic liposarcoma & $6(6.45 \%)$ & 0 \\
\hline $\begin{array}{l}\text { Pleomorphic } \\
\text { rhabdomyosarcoma }\end{array}$ & $5(5.38 \%)$ & $1(1.3 \%)$ \\
\hline $\begin{array}{l}\text { Undifferentiated pleomorphic } \\
\text { sarcoma }\end{array}$ & $31(33.33 \%)$ & $20(25.97 \%)$ \\
\hline
\end{tabular}

Numbers in brackets are $95 \%$ confidence intervals. Other histological subtypes in the sporadic cohort are: two malignant solitary fibrous tumors, one synovial sarcoma, and one low-grade fibromyxoid sarcoma. Other histological subtypes in the post-radiation cohort are: four undifferentiated spindle cell sarcomas, three extraskeletal osteosarcomas, two malignant mesenchymomas, one intimal sarcoma, one malignant peripheral nerve sheath tumor, and one osteosarcoma

which patients had previously reported cancers. Gene Expression Omnibus (GEO) accession: GSE71119 and Sequence Read Archive (SRA) accession: SRP057793. Data for the 77 post-radiation sarcomas are available at GEO accession: GSE102055 and SRA accession: SRP113755.

\section{Results}

\section{Genetic specificity of post-radiation angiosarcomas}

As previously reported, we observed a high frequency of MYC amplifications in all but one (96\%) post-radiation 
angiosarcomas. Besides MYC alterations, there were a few other genomic alterations (Sup. Fig. 1). Since recurrent $K D R$ (coding for the VEGFR-2 protein) mutations were also reported in post-radiation angiosarcomas, we screened variations of this gene in our 24 cases. Two of them (8\%) harbor missense $K D R$ variants: p.S691C (NM_002253 exon 14, c.2072C $>$ G; case AA6201; Sup. Fig. 2A) and p. D1312H (NM_002253 exon 30, c.3934G >C; case AA6175; Sup. Fig. 2B). These two variations involve the extracellular and cytoplasmic regions of the VEGFR-2 protein, respectively. To date, no domain has been reported for D1312 while S691 is located in the immunoglobulin-like C2-type 7 domain, which has not been identified as an essential component for VEGF-A or VEGF-C interaction [30].

\section{Genomic characterization of post-radiation and sporadic sarcomas with complex genetics}

To characterize genomic alterations in these groups, we performed DNA-arrays on 11 post-radiation leiomyosarcomas and 20 post-radiation undifferentiated pleomorphic sarcomas and compared the profiles to those of 34 sporadic leiomyosarcomas and 22 sporadic undifferentiated pleomorphic sarcomas (respectively, including 7 and 5 cases from the reference cohort of 93 sporadic tumors analyzed by RNA-seq). We considered both gains and losses observed in the different groups (leiomyosarcomas vs. undifferentiated pleomorphic sarcomas and/or sporadic vs. post-radiation sarcomas) with penetrance plots (Fig. 1). Globally, sporadic and post-radiation sarcomas had the same levels of genomic alterations, including those that typically occur in sarcomas with complex genetics: losses of chromosomes 10, 13, 16 and gains of chromosomes 1, 9, 14 with high frequencies [31-33]. In particular, RBI (13q14.2) was targeted at the same frequency in both sporadic and post-radiation tumors (nearly $75 \%$ for sporadic and post-radiation leiomyosarcomas; $85 \%$ and $75 \%$ for sporadic and post-radiation undifferentiated pleomorphic sarcomas, respectively; Sup. Fig. 3: upper panel), in agreement with the literature.

However, a difference can be observed in the 9p21.3 region (including $C D K N 2 A$ and $C D K N 2 B$, coding for p14/ p16 and p15 proteins, respectively; Sup. Fig. 3: lower panel). This region was lost in $35 \%$ of sporadic leiomyosarcomas vs. $64 \%$ of post-radiation ones and in $45 \%$ of sporadic undifferentiated pleomorphic sarcomas vs. $75 \%$ of post-radiation ones. Grouped together (leiomyosarcomas and undifferentiated pleomorphic sarcomas), this region was significantly lost with a higher frequency in postradiation than in sporadic sarcomas $(71 \%$ and $39 \%$, respectively; Fisher's $P=6.92 \mathrm{e}-3$ ). In sporadic tumors, $C D K N 2 A$ loss co-occurred with $R B 1$ loss in $86 \%$ of tumors (19 cases out of 22). In post-radiation tumors, this intersection was lower and represented $68 \%$ of cases (15 cases out of 22; binomial probability $P(X \leq 15)=2.3 \mathrm{e}-2)$, while 7 tumors out of 31 (23\%) lost $C D K N 2 A$ but not $R B 1$. Taken together, $R B 1$ or $C D K N 2 A$ losses occurred in $91 \%$ of sporadic sarcomas (48 cases out of 53 ) and $97 \%$ of postradiation ones (30 cases out of 31 ).

\section{Mutational specificity of post-radiation sarcomas}

An initial pool of 310,641 unfiltered variants was detected in all the available cases processed by RNA-seq: 93 sporadic and 77 post-radiation sarcomas (see the section "Material and methods"). After the filtration step, we obtained 5594 variants covering 3925 genes.

For each gene, we reported the number of altered sporadic and post-radiation tumors. Then, gene variations having an incidence difference of $<5 \%$ in the two cohorts were removed. A total of 75 variations corresponding to 21 genes was identified with minimum and maximum incidence differences of $5.19 \%$ and $11.38 \%$, respectively (Table 2). Among these 21 genes, MUTYH was the only one reported in the Cancer Gene Census database (2018/03 version) [34] in the context of colon cancer as an alternative to $A P C$ mutations in familial adenomatous polyposis. Though no variant was detected for this gene in sporadic sarcomas, four missense variants were detected in postradiation ones (cases: AA6216, AA6209, AA6233, and AA6182) in different exons $(16,13,11$, and 10, respectively; NM_001048171 transcript). These sarcomas were, respectively, two undifferentiated pleomorphic sarcomas, one extraskeletal osteosarcoma and one undifferentiated spindle cell sarcoma. Their respective locations were the vagina, chest wall, shoulder girdle, and orbit.

We then looked for differences in mutational patterns on filtered variants depending on the radiation context. First, no difference in terms of mutation types (missense, nonsense, and stop loss) or base substitutions (transversion or transitions) was detected (Fisher's $P$-values are $1.86 \mathrm{e}-1$ and $1 \mathrm{e}-1$, respectively). Second, taking into account the nucleotides before and after the variant one, we obtained similar profiles between sporadic and post-radiation tumors, corresponding to the spontaneous deamination of the 5methyl-cytosine signature. This mutational signature has been observed in many cancer types and represents an aging signature, since this phenomenon constitutionally occurs in lifetime [35].

\section{Fusion genes in secondary sarcomas}

A total of 25,142 and 9163 fusion genes was predicted for sporadic and post-radiation sarcomas, respectively (see the section "Material and methods"). After the filtration step, 2480 and 515 fusion genes were investigated in these groups, respectively. Among the 515 fusion genes detected 


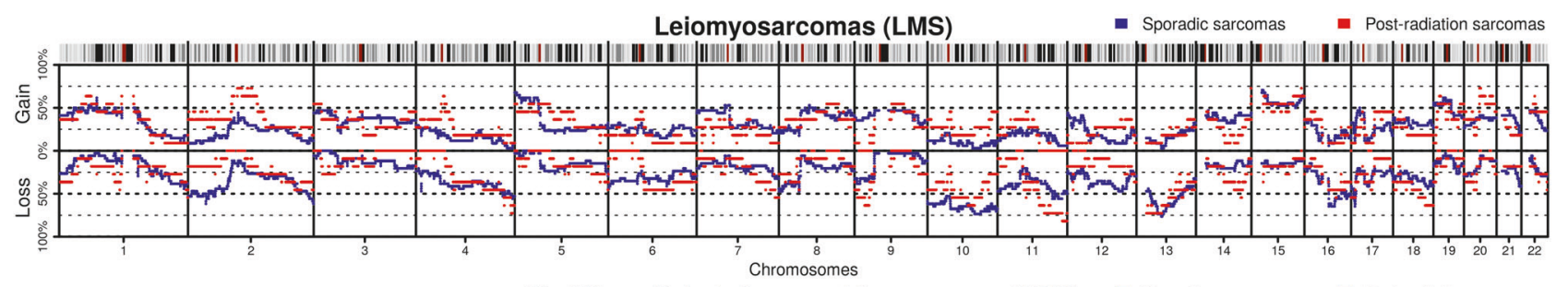

Undifferentiated pleomorphic sarcomas (UPS) - Sporadic sarcomas - Post-radiation sarcomas

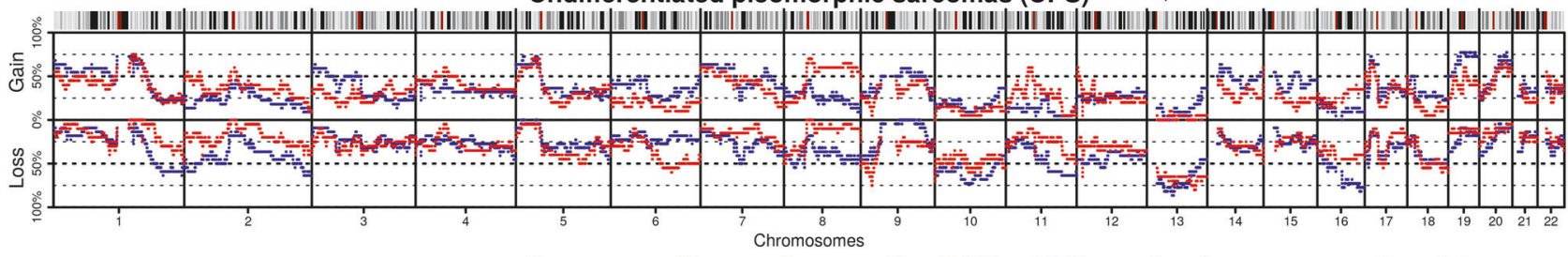

Sarcomas with complex genetics (LMS + UPS) - Sporadic sarcomas

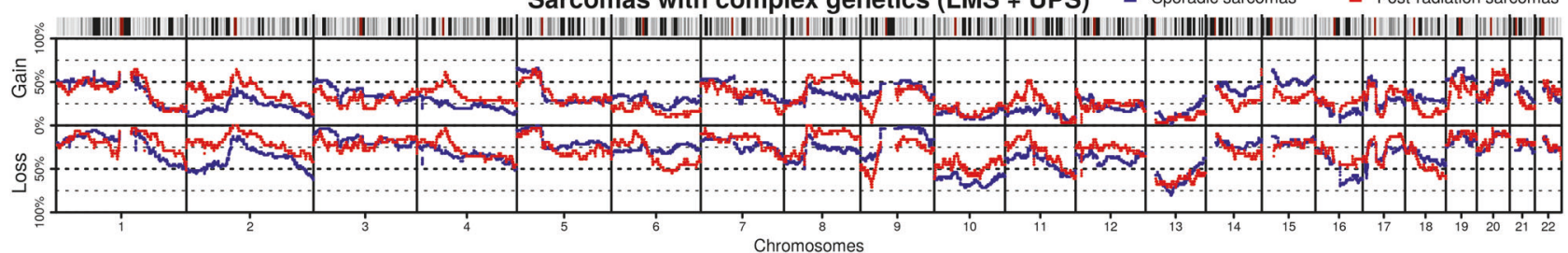

Fig. 1 Penetrance plots showing recurrent copy number variations in leiomyosarcomas (LMS; upper panel: 34 sporadic and 11 postradiation tumors), undifferentiated pleomorphic sarcomas (UPS; intermediate panel: 22 sporadic and 20 post-radiation tumors), and pleomorphic sarcomas (LMS + UPS; lower panel). Each panel displays gains upwards and losses downwards for chromosomes 1-22 and includes cytogenetic bands on top in post-radiation sarcomas, we kept those that recurred in this group (possibly with different partners) and were not detected in sporadic sarcomas. Twelve genes fulfilled these conditions but sequence reanalysis excluded 10 of them as false-positives due to sequence homology. Two genes were subsequently investigated by in silico analyses: RUFY1 and TRAM1. RUFY1 is either fused with CMTM6 $\left(C^{2} M T M 6_{\text {exon }}\right.$ ${ }_{1}-R U F Y 1_{\text {exon } 12} ; \mathrm{t}(3 ; 5)$; case AA6176) or deleted (RUFY $1_{5^{\prime}}$ UTR-intergenic; case AA6206). TRAM1 is either fused with RAVER1 (TRAM1 $1_{\text {exon }} 10-R A V E R 1_{\text {exon }} 1 ; \mathrm{t}(8 ; 19) ; \quad$ case AA6218) or produces an inter-chromosomal transcript

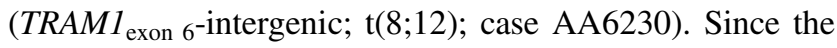
partners were not identical and each concerned only two cases, a fusion gene was ruled out as a main mechanism for the oncogenesis of post-radiation sarcomas compared to sporadic ones.

\section{Transcriptome analysis of post-radiation vs. sporadic sarcomas}

To analyze homogeneous sub-groups and maximize transcriptomic differences, we focused on leiomyosarcomas and undifferentiated pleomorphic sarcomas. Unsupervised methods ( $\mathrm{t}-\mathrm{SNE}$ and clustering) comparing sporadic (22 leiomyosarcomas plus 31 undifferentiated pleomorphic sarcomas) and post-radiation (11 leiomyosarcomas plus 20 undifferentiated pleomorphic sarcomas) sarcomas failed to identify any transcriptomic differences, suggesting that these groups share similar transcriptomic patterns (Fig. 2). In addition, the seven published gene expression signatures related to irradiation failed to categorize sporadic and postradiation tumors (Fisher's $P$ with Benjamini-Hochberg adjustment $>0.05$; Sup. Fig. 4).

Supervised differential expression analysis between sporadic and post-radiation sarcomas identified 529 upregulated genes in sporadic tumors and 198 in postradiation tumors (Sup. Tables 1 and 2). The main gene ontology associated with sporadic tumors is cation transmembrane transport $(Q=9.9 \mathrm{e}-3)$. The main gene ontologies associated with post-radiation tumors are responses to oxygen-containing compounds and organic substances $(Q$ are $3.19 \mathrm{e}-5$ and $2.73 \mathrm{e}-4$, respectively), positive regulation of lipid storage $(Q=8.36 \mathrm{e}-3)$ and inflammatory response $(Q=1.28 \mathrm{e}-2)$. No enrichments for response to radiation ontology (or associated sub-terms) were detected.

\section{Characterization of secondary angiosarcomas}

We then analyzed the entire cohort of secondary sarcomas, including diverse histological subtypes (Table 1), but excluding sporadic tumors. Though no transcriptomic pattern was measured in almost all post-radiation tumors, angiosarcomas carried a distinct gene expression profile: all of the 24 cases clustered together (Fig. 3). Accordingly, we 
Table 2 Genes for which variants were detected in postradiation and sporadic sarcomas with a difference in incidence of more than $5 \%$

\begin{tabular}{|c|c|c|c|c|c|c|c|c|}
\hline \multirow[t]{2}{*}{ Gene } & \multicolumn{2}{|c|}{$\begin{array}{l}\text { Variants in } \\
\text { post-radiation } \\
\text { sarcomas }\end{array}$} & \multicolumn{2}{|c|}{$\begin{array}{l}\text { Variants in } \\
\text { sporadic } \\
\text { sarcomas }\end{array}$} & \multicolumn{2}{|c|}{$\begin{array}{l}\text { Absolute } \\
\text { difference }\end{array}$} & \multirow[t]{2}{*}{ Association } & \multirow[t]{2}{*}{ CGC } \\
\hline & $n$ & $\%$ & $n$ & $\%$ & $n$ & $\%$ & & \\
\hline$N B P F 12$ & 2 & 2.6 & 13 & 13.98 & 11 & 11.38 & Sporadic & No \\
\hline$N B P F 11$ & 5 & 6.49 & 14 & 15.05 & 9 & 8.56 & Sporadic & No \\
\hline$A N A P C 1$ & 3 & 3.9 & 11 & 11.83 & 8 & 7.93 & Sporadic & No \\
\hline RASA4 & 1 & 1.3 & 7 & 7.53 & 6 & 6.23 & Sporadic & No \\
\hline$R A S A 4 B$ & 1 & 1.3 & 7 & 7.53 & 6 & 6.23 & Sporadic & No \\
\hline$B O P 1$ & 1 & 1.3 & 7 & 7.53 & 6 & 6.23 & Sporadic & No \\
\hline$G T F 2 H 2 C$ & 0 & 0 & 5 & 5.38 & 5 & 5.38 & Sporadic & No \\
\hline GTF2IRD2 & 0 & 0 & 5 & 5.38 & 5 & 5.38 & Sporadic & No \\
\hline MUTYH & 4 & 5.19 & 0 & 0 & 4 & 5.19 & Post-radiation & Yes \\
\hline ZFP62 & 4 & 5.19 & 0 & 0 & 4 & 5.19 & Post-radiation & No \\
\hline$P D C D 11$ & 4 & 5.19 & 0 & 0 & 4 & 5.19 & Post-radiation & No \\
\hline$U B E 4 A$ & 4 & 5.19 & 0 & 0 & 4 & 5.19 & Post-radiation & No \\
\hline$C D C 16$ & 4 & 5.19 & 0 & 0 & 4 & 5.19 & Post-radiation & No \\
\hline$U L K 3$ & 4 & 5.19 & 0 & 0 & 4 & 5.19 & Post-radiation & No \\
\hline$P L X N B 2$ & 4 & 5.19 & 0 & 0 & 4 & 5.19 & Post-radiation & No \\
\hline$D D T$ & 7 & 9.09 & 3 & 3.23 & 4 & 5.87 & Post-radiation & No \\
\hline$A B C B 10$ & 5 & 6.49 & 0 & 0 & 5 & 6.49 & Post-radiation & No \\
\hline $\mathrm{MROH} 1$ & 15 & 19.48 & 10 & 10.75 & 5 & 8.73 & Post-radiation & No \\
\hline$C B W D 3$ & 10 & 12.99 & 3 & 3.23 & 7 & 9.76 & Post-radiation & No \\
\hline TMEM68 & 8 & 10.39 & 0 & 0 & 8 & 10.39 & Post-radiation & No \\
\hline$E P B 41 L 3$ & 8 & 10.39 & 0 & 0 & 8 & 10.39 & Post-radiation & No \\
\hline
\end{tabular}

$C G C$ the Cancer Gene Census database
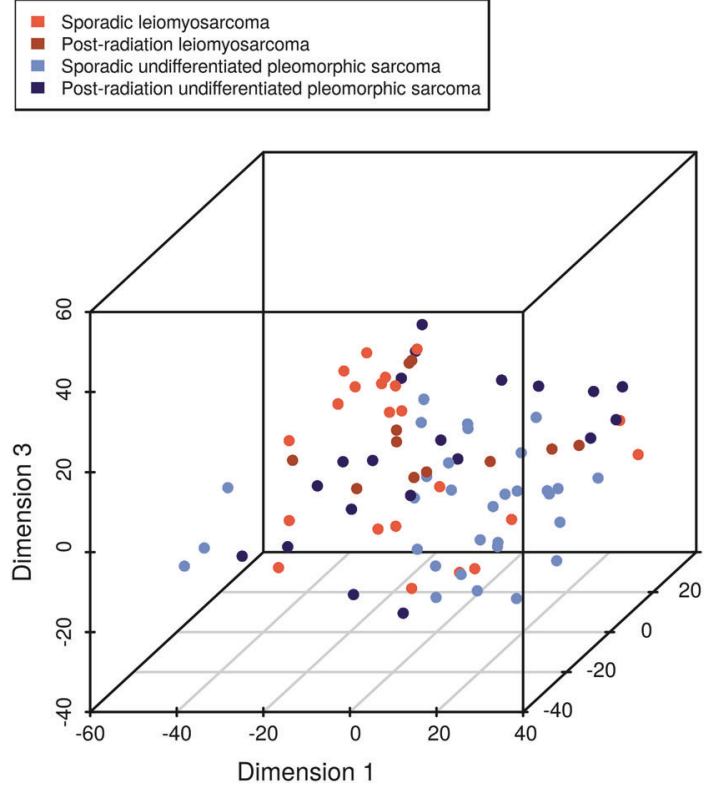

Fig. 2 unsupervised clustering methods on pleomorphic sporadic sarcomas and post-radiation ones (t-SNE and agglomerative hierarchical clustering in the left and right panels, respectively) to segregate tumors

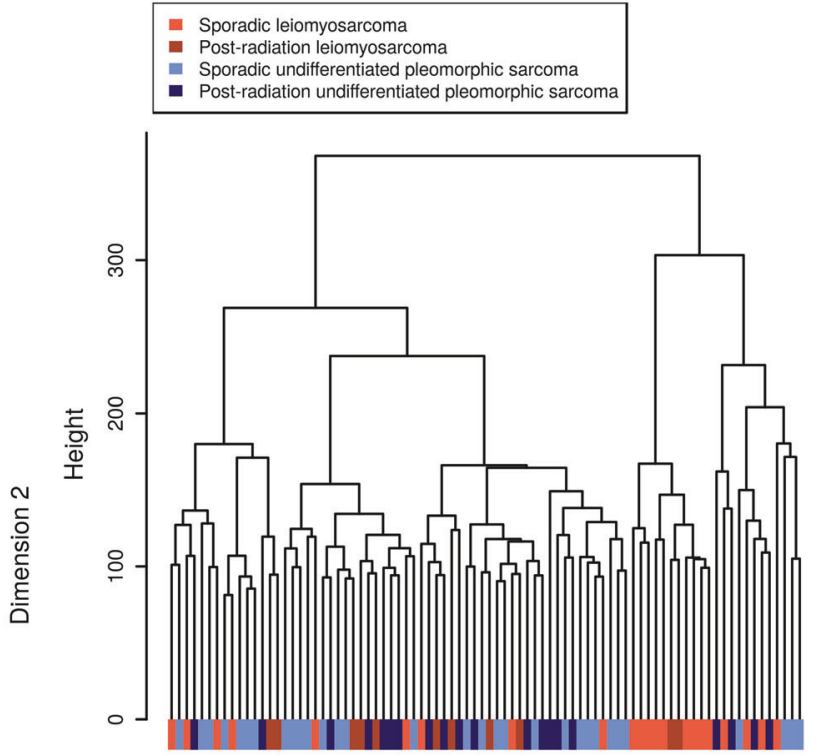

based on their transcriptomic profiles. This shows a mixed stratification for these groups, so they share global transcriptomic similarities 

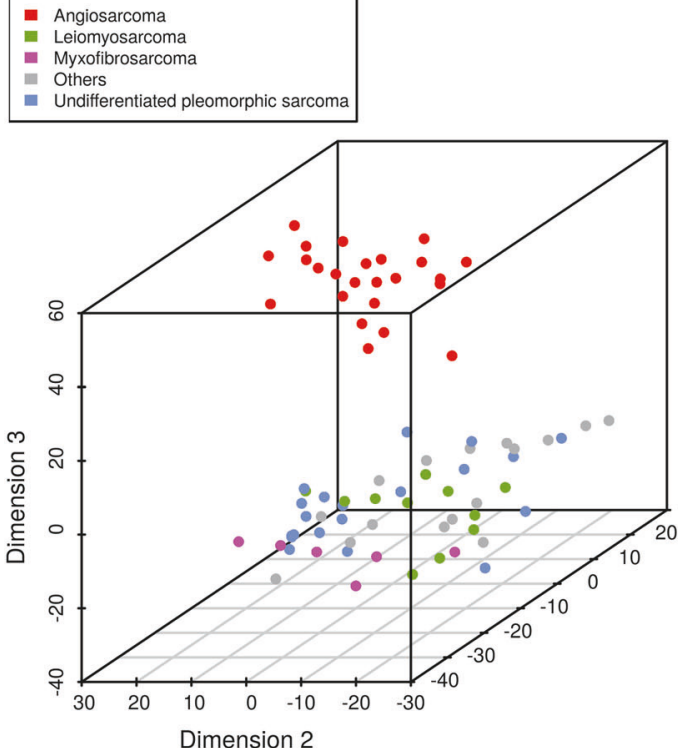

Fig. 3 unsupervised clustering methods on all available post-radiation sarcomas (t-SNE and principal component analysis in the left and right panels, respectively) to segregate tumors based on their transcriptomic

performed gene set enrichment analysis on known $M Y C$ targets and observed that they were up-regulated in angiosarcomas but not in other types $(P=1.5 \mathrm{e}-2$; Sup. Fig. 5).

These 24 angiosarcomas also displayed two different transcriptomic patterns, corresponding to a first group of eight tumors termed ASg1 and a second group of 16 tumors termed ASg2 (Fig. 3). Though 75\% of patients in ASg1 died (6 among 8) compared to $37.5 \%$ in ASg2 (6 among $16)$, these overall survivals were not significantly distinct $(P=1.09 \mathrm{e}-1$; hazard ratio $=2.57[0.78-8.49])$, and the two groups also shared similar clinical characteristics (vital status, age at diagnosis, tumor size, site, cell differentiation, necrosis, number of mitosis, and grade of tumors; all $P>$ 0.05; Sup. Table 3). Supervised differential expression analysis between the two groups identified 1554 upregulated genes in ASg1 and 2211 in ASg2 (Sup. Tables 4 and 5). The main gene ontology associated with ASg1 was protein translation (and associated sub-terms; $Q<1 \mathrm{e}-10)$ through the overexpression of most $(71 \%)$ of the ribosomal proteins (both small and large subunits, including mitochondrial ribosomal proteins). The main gene ontologies associated with $\mathrm{ASg} 2$ were cell adhesion, immune response, and regulation of cell communication and migration (all $Q<1 \mathrm{e}-10$ ). MYC was not differentially expressed between $\mathrm{ASg} 1$ and $\mathrm{ASg} 2(P=7.03 \mathrm{e}-1 ; \log 2-$ fold change $=-0.19$ ).

The gene ontologies associated with $\mathrm{ASg} 2$ conducted an advanced immune characterization. We took advantages of the CIBERSORT approach (RNA-seq-based) to estimate immune populations in the post-radiation angiosarcomas

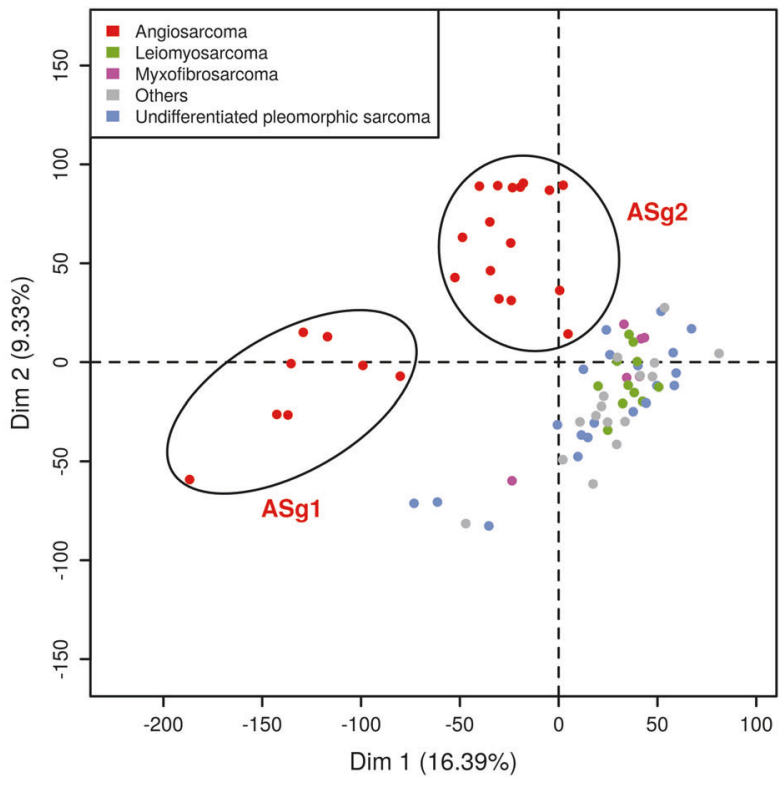

profiles. This shows a distinct transcriptomic pattern for angiosarcomas compared to other histological subtypes. Principal component analysis highlights two angiosarcoma sub-groups, termed ASg1 and ASg2

[36]. Among the 22 immune populations analyzed by CIBERSORT, none was significantly different between ASg1 and ASg2 (Sup. Fig. 6). We consequently studied the immune infiltrate at the cellular level with histochemistry (Sup. Table 3). The ASg2 group was characterized by a higher nuclear atypia $(P=1.08 \mathrm{e}-2)$, a higher intensity of inflammation $(P=2.45 \mathrm{e}-2)$, and a higher immune infiltrate at tumor periphery $(P=3.95 \mathrm{e}-3)$. No significant difference was observed with the immune infiltrate neither in the tumor stroma nor in direct contact with tumor cells. Finally, we characterized the immune infiltrate at the cell type level.

From a chromosomal standpoint, these two groups had few genomic imbalances except chromosome 17q24.217qter, which was significantly gained in 12 cases (75\%) of ASg2 vs. two cases (25\%) of ASg1 (Fisher's $P=3.24 \mathrm{e}-2$; Sup. Fig. 7). This specific genomic disorder did not modify the gene expression in this region (among 212 listed protein-coding genes, 21 and 23 were overexpressed in $\mathrm{ASg} 1$ and $\mathrm{ASg} 2$, respectively).

\section{Discussion}

Post-radiation sarcomas represent a clinical challenging group of tumors. They are very aggressive neoplasms with few effective therapeutic opportunities. However, identifying some recurrent molecular characteristics holds promise for effective targeted therapy. For example, as KDR encodes one of the VEGF receptors, $K D R$-altered post-radiation angiosarcomas might be more sensitive to tyrosine kinase 
inhibitors like sunitinib or sorafenib compared to wild-type KDR tumors [18]. Nevertheless, a better understanding of cancer genomics is needed, particularly in sarcomas with complex genetics, associated with massive genome reshuffling and misunderstood oncogenesis [37]. We thus performed a genome-wide characterization of post-radiation sarcomas and correlated it to consequences on their transcriptomes to better understand the biology of these tumors.

In sarcomas with complex genetics, we observed that sporadic and post-radiation sarcomas share similar genetic alterations, including $R B 1$, which is lost in nearly $75 \%$ of all such tumors. Interestingly, $C D K N 2 A$ and $C D K N 2 B$ are significantly lost with a higher frequency in post-radiation than in sporadic sarcomas $(71 \%$ and $39 \%$, respectively; Fisher's $P=6.92 \mathrm{e}-3)$. The p16 protein $(C D K N 2 A)$ inhibits the cyclin $\mathrm{D} / \mathrm{Cdk} 4$ complex (cycle cell pathway) that phosphorylates $\mathrm{pRb}(R B 1)$, allowing cells to enter the $\mathrm{S}$ phase [38]. Consequently, $C D K N 2 A$ inactivation could replace $R B 1$ loss in post-radiation sarcomas, since all but one tumor harbored at least one of the two alterations, so it might play a role in the oncogenesis of such tumors by destabilizing the cycle cell progression.

In sarcomas with complex genetics, we did not observe any specific transcriptomic pattern in post-radiation sarcomas compared to sporadic ones. First, no recurrent and driver fusion genes have been characterized in these two groups [37, 39]. Second, a supervised approach with differential gene expression analyses did not highlight a strong oncogenic pathway in either of the two cohorts. Third, unsupervised approaches with clustering and t-SNE were unable to categorize whole transcriptome profiles either in terms of radiation context or the seven radiation-related signatures [22-28]. However, owing to technical considerations, the two latter points require clarification, as we selected non-overlapping coding genes owing to a difference in the sequencing strategy (see the section "Material and methods"). Nevertheless, more than 16,500 (87\%) protein-coding genes were considered in these analyses, without any evidence of batch effects.

We validated the previously described alterations in postradiation angiosarcomas in our independent cohort. Two cases $(8 \%)$ presented $K D R$ variants, in accordance with the reported 10\% [18]. All but one case (96\%) presented MYC amplifications, in accordance with the reported high frequencies $[14,17]$. In the two groups of post-radiation angiosarcomas, no association was found with clinical characteristics. Though the overall survival analysis was non-significant $(P=1.09 \mathrm{e}-1)$, probably due to the cohort size, $\mathrm{ASg} 1$ were highly aggressive sarcomas (6 out of 8 patients died; $25 \%$ overall survival) compared to $\mathrm{ASg} 2$ (6 out of 16 patients died; $62.5 \%$ overall survival). The transcriptomic difference between these two groups led us to characterize the immune infiltrate.
The CIBERSORT approach did not identify differences in terms of cell populations. However, this result is probably biased because of a lack of immune cells within the tumor stroma of both groups. Instead, histochemistry revealed a higher intensity of inflammation and a higher immune infiltrate at tumor periphery $(P$ are $2.45 \mathrm{e}-2$ and $3.95 \mathrm{e}-2$, respectively) in $\mathrm{ASg} 2$, the least aggressive group. Consequently, in these two groups of post-radiation angiosarcomas, the involvement of the immune system could explain the differences in terms of vital status. Because of a lack of statistical power to accurately measure the contribution of the immune system to the clinical course, a larger cohort may provide a better understanding of such sub-groups.

DNA-array is a useful technique for understanding cancer genomics [40]. Particularly, high-resolution arrays, such as the CytoScan HD (Affymetrix) used in this analysis can identify gains and losses of a few kilobases. Whole genome sequencing (DNA-seq) offers the opportunity to precisely detect a wide spectrum of genomic alterations, being able to work at a single base pair resolution. We did not observe a mutational signature specific to post-radiation sarcomas using RNA-seq, so coding point mutations are likely either secondary events not depending on oncogenesis initiation mechanisms, or these mechanisms are identical between sporadic tumors and post-radiation ones. This assumption should nevertheless be taken with caution, since our analysis was restricted to point variants (all of them are not actual cancer mutations) in expressed genes (whereas DNA-seq covers the whole genome). Mutational signatures have been identified in secondary malignancies, namely angiosarcomas, osteosarcomas, spindle cell sarcomas, and breast tumors [41]. These tumors $(n=12)$, compared to a reference cohort of primary cancers $(n=319)$, significantly harbored more deletions relative to insertions and balanced inversions. Consequently, DNA-seq is a promising tool to better understand consequences of radiations in the context of cancer therapy by measuring changes in DNA that may precise their oncogenesis.

Radiation therapies cause DNA damage to normal cells surrounding tumor cells. It is tempting to postulate that such DNA damage may directly alter oncogenic drivers and lead to cancer development. However, secondary tumors rarely arise after such frequently administered treatment. Thus, most DNA damage may be passenger mutations while the driver ones may occur after the cell enters an unstable state. In this context, constitutive variants that allow oncogenic alterations to occur would be good candidates for promoting tumor initiation. A constitutive study of variants found in two groups of patients with and without secondary tumors, and using DNA or exome sequencing would answer this point. 
Acknowledgements Computer time was provided by the MCIAMésocentre de Calcul Intensif Aquitain (MCIA) computing facility at the University of Bordeaux and the University of Pau and Pays de l'Adour. We are grateful to the French Sarcoma Group for tumor banks and associated clinical annotations. The following French cancer centers participated in this study: Institut Bergonié (Bordeaux), Centre Georges-François Leclerc (Dijon), Hôpital Pitié-Salpetrière (Paris), Centre Paul Papin (Angers), Centre François Baclesse (Caen), Centre Jean Perrin (Clermont-Ferrand), Centre Alexis Vautrin (Nancy), Institut Curie (Paris), Institut Gustave Roussy (Villejuif), Centre Oscar Lambert (Lille), Centre Léon Bérard (Lyon), Institut du Cancer de Montpellier (Montpellier), Centre Antoine Lacassagne (Nice), Institut Universitaire du Cancer de Toulouse (Toulouse), and Centre Hospitalier Régional Universitaire de Tours (Tours).

Funding This project was financially supported by the PHRC 2011 program (PHRC11-102) and Inserm.

\section{Compliance with ethical standards}

Conflict of interest The authors declare that they have no conflict of interest.

Publisher's note: Springer Nature remains neutral with regard to jurisdictional claims in published maps and institutional affiliations.

\section{References}

1. Brady MS, Gaynor JJ, Brennan MF. Radiation-associated sarcoma of bone and soft tissue. Arch Surg. 1992;127:1379-85.

2. Mark RJ, Poen J, Tran LM, et al. Postirradiation sarcomas. A single-institution study and review of the literature. Cancer. 1994;73:2653-62.

3. Cha C, Antonescu CR, Quan ML, et al. Long-term results with resection of radiation-induced soft tissue sarcomas. Ann Surg. 2004;239:903-9. discussion 909-10.

4. Cahan WG, Woodard HQ, Higinbotham NL, et al. Sarcoma arising in irradiated bone: report of 11 cases. Cancer. 1948;1:3-29.

5. Arlen M, Higinbotham NL, Huvos AG, et al. Radiation-induced sarcoma of bone. Cancer. 1971;28:1087-99.

6. Gladdy RA, Qin L-X, Moraco N, et al. Do radiation-associated soft tissue sarcomas have the same prognosis as sporadic soft tissue sarcomas? J Clin Oncol. 2010;28:2064-9.

7. Maddams J, Parkin DM, Darby SC. The cancer burden in the United Kingdom in 2007 due to radiotherapy. Int J Cancer. 2011;129:2885-93.

8. Wiklund TA, Blomqvist CP, Räty J, et al. Postirradiation sarcoma. Analysis of a nationwide cancer registry material. Cancer. 1991;68:524-31.

9. Lagrange JL, Ramaioli A, Chateau MC, et al. Sarcoma after radiation therapy: retrospective multiinstitutional study of 80 histologically confirmed cases. Radiation Therapist and Pathologist Groups of the Fédération Nationale des Centres de Lutte Contre le Cancer. Radiology. 2000;216:197-205.

10. Bjerkehagen B, Smeland S, Walberg L, et al. Radiation-induced sarcoma: 25-year experience from the Norwegian Radium Hospital. Acta Oncol. 2008;47:1475-82.

11. Riad S, Biau D, Holt GE, et al. The clinical and functional outcome for patients with radiation-induced soft tissue sarcoma. Cancer. 2012;118:2682-92.

12. Sheppard DG, Libshitz HI. Post-radiation sarcomas: a review of the clinical and imaging features in 63 cases. Clin Radio. 2001;56:22-29.
13. Bjerkehagen $\mathrm{B}$, Småstuen $\mathrm{MC}$, Hall $\mathrm{KS}$, et al. Why do patients with radiation-induced sarcomas have a poor sarcoma-related survival? Br J Cancer. 2012;106:297-306.

14. Käcker C, Marx A, Mössinger K, et al. High frequency of MYC gene amplification is a common feature of radiation-induced sarcomas. Further results from EORTC STBSG TL 01/01. Genes Chromosomes Cancer. 2013;52:93-98.

15. Ravanat J-L, Breton J, Douki T, et al. Radiation-mediated formation of complex damage to DNA: a chemical aspect overview. Br J Radio. 2014;87:20130715.

16. Kuttesch JF, Wexler LH, Marcus RB, et al. Second malignancies after Ewing's sarcoma: radiation dose-dependency of secondary sarcomas. J Clin Oncol. 1996;14:2818-25.

17. Manner J, Radlwimmer B, Hohenberger P, et al. MYC high level gene amplification is a distinctive feature of angiosarcomas after irradiation or chronic lymphedema. Am J Pathol. 2010;176:34-39.

18. Antonescu CR, Yoshida A, Guo T, et al. KDR activating mutations in human angiosarcomas are sensitive to specific kinase inhibitors. Cancer Res. 2009;69:7175-9.

19. Chibon F, Mairal A, Fréneaux P, et al. The RB1 gene is the target of chromosome 13 deletions in malignant fibrous histiocytoma. Cancer Res. 2000;60:6339-45.

20. Gonin-Laurent N, Hadj-Hamou NS, Vogt N, et al. RB1 and TP53 pathways in radiation-induced sarcomas. Oncogene. 2007;26:6106-12.

21. Pérot G, Chibon F, Montero A, et al. Constant p53 pathway inactivation in a large series of soft tissue sarcomas with complex genetics. Am J Pathol. 2010;177:2080-90.

22. Hadj-Hamou N-S, Ugolin N, Ory C, et al. A transcriptome signature distinguished sporadic from postradiotherapy radiationinduced sarcomas. Carcinogenesis. 2011;32:929-34.

23. Tsai M-H, Chen X, Chandramouli GVR, et al. Transcriptional responses to ionizing radiation reveal that $\mathrm{p} 53 \mathrm{R} 2$ protects against radiation-induced mutagenesis in human lymphoblastoid cells. Oncogene. 2006;25:622-32.

24. Zhou T, Chou J, Mullen TE, et al. Identification of primary transcriptional regulation of cell cycle-regulated genes upon DNA damage. Cell Cycle. 2007;6:972-81.

25. Amundson SA, Do KT, Vinikoor LC, et al. Integrating global gene expression and radiation survival parameters across the 60 cell lines of the National Cancer Institute Anticancer Drug Screen. Cancer Res. 2008;68:415-24.

26. Ghandhi SA, Yaghoubian B, Amundson SA. Global gene expression analyses of bystander and alpha particle irradiated normal human lung fibroblasts: synchronous and differential responses. BMC Med Genom. 2008;1:63.

27. Warters RL, Packard AT, Kramer GF, et al. Differential gene expression in primary human skin keratinocytes and fibroblasts in response to ionizing radiation. Radiat Res. 2009;172:82-95.

28. Smirnov DA, Brady L, Halasa K, et al. Genetic variation in radiation-induced cell death. Genome Res. 2012;22:332-9.

29. Lesluyes T, Pérot G, Largeau MR, et al. RNA sequencing validation of the Complexity INdex in SARComas prognostic signature. Eur J Cancer. 2016;57:104-11.

30. Leppänen V-M, Prota AE, Jeltsch M, et al. Structural determinants of growth factor binding and specificity by VEGF receptor 2. Proc Natl Acad Sci USA. 2010;107:2425-30.

31. Chibon F, Mariani O, Mairal A, et al. The use of clustering software for the classification of comparative genomic hybridization data. an analysis of 109 malignant fibrous histiocytomas. Cancer Genet Cytogenet. 2003;141:75-78.

32. Guillou L, Aurias A. Soft tissue sarcomas with complex genomic profiles. Virchows Arch. 2010;456:201-17.

33. Gibault L, Pérot G, Chibon F, et al. New insights in sarcoma oncogenesis: a comprehensive analysis of a large series of $160 \mathrm{soft}$ tissue sarcomas with complex genomics. J Pathol. 2011;223:64-71. 
34. Futreal PA, Coin L, Marshall M, et al. A census of human cancer genes. Nat Rev Cancer. 2004;4:177-83.

35. Alexandrov LB, Nik-Zainal S, Wedge DC, et al. Signatures of mutational processes in human cancer. Nature. 2013;500: 415-21.

36. Newman AM, Liu CL, Green MR, et al. Robust enumeration of cell subsets from tissue expression profiles. Nat Methods. 2015;12:453-7.

37. The Cancer Genome Atlas Research Network. Comprehensive and integrated genomic characterization of adult soft tissue sarcomas. Cell. 2017;171:950-.e28.
38. Nielsen GP, Burns KL, Rosenberg AE, et al. CDKN2A gene deletions and loss of p16 expression occur in osteosarcomas that lack RB alterations. Am J Pathol. 1998;153:159-63.

39. Delespaul L, Lesluyes T, Pérot G, et al. Recurrent TRIO fusion in nontranslocation-related sarcomas. Clin Cancer Res. 2017;23:857-67.

40. Bejjani BA, Shaffer LG. Application of array-based comparative genomic hybridization to clinical diagnostics. J Mol Diagn. 2006;8:528-33.

41. Behjati S, Gundem G, Wedge DC, et al. Mutational signatures of ionizing radiation in second malignancies. Nat Commun. 2016;7:12605.

\section{Affiliations}

Tom Lesluyes $\mathbb{D}^{1,2,3,4} \cdot$ Jessica Baud ${ }^{1,2} \cdot$ Gaëlle Pérot $^{1,5} \cdot$ Céline Charon-Barra $^{6} \cdot$ Axel You $^{1,7} \cdot$ Isabelle Valo ${ }^{8}$. Céline Bazille $^{9}$ - Florence Mishellany ${ }^{10}$ - Agnès Leroux ${ }^{11}$. Sophie Renard-Oldrini ${ }^{12}$. Philippe Terrier $\mathbb{D}^{13}$. Axel Le Cesne ${ }^{14}$. Marick Laé ${ }^{15,16}$. Sophie Piperno-Neumann ${ }^{17}$. Sylvie Bonvalot $\mathbb{D}^{18}$. Agnès Neuville ${ }^{5,20,20}$. Françoise Collin $^{6} \cdot$ Philippe Maingon $^{19}$ • Jean-Michel Coindre ${ }^{2,5}$ • Frédéric Chibon $\mathbb{1}^{3,4}$

Inserm U1218, Institut Bergonié, Bordeaux, France

2 University of Bordeaux, F-33000 Bordeaux, France

3 Inserm UMR1037, Cancer Research Center of Toulouse, Toulouse, France

4 Institut Claudius Regaud, IUCT-Oncopole, Toulouse, France

5 Department of Pathology, Institut Bergonié, Bordeaux, France

6 Department of Pathology, Centre Georges-François Leclerc, Dijon, France

7 University of Nantes, F-44000 Nantes, France

8 Department of Pathology, Institut de cancérologie de l'Ouest site Paul Papin, Angers, France

9 Department of Pathology, University Hospital, Caen, France

10 Department of Pathology, Centre Jean Perrin, ClermontFerrand, France

11 Department of Pathology, Centre Alexis Vautrin, Vandoeuvre-lèsNancy, France
12 Department of Radiation Therapy, Centre Alexis Vautrin, Vandoeuvre-lès-Nancy, France

13 Department of Pathology, Institut Gustave Roussy, Villejuif, France

14 Department of Medical Oncology, Institut Gustave Roussy, Villejuif, France

15 Department of Pathology, Institut Curie, Paris, France

16 Department of Pathology, Centre Henri Becquerel, Inserm U1245, UniRouen Normandy University, Rouen, France

17 Department of Medical Oncology, Institut Curie, Paris, France

18 Department of Surgery, Institut Curie, Paris, France

19 Department of Radiation Oncology, Hôpital La Pitié-Salpêtrière, Sorbonne University, Paris, France

20 Present address: Contades Office of Pathological Anatomy and Cytology, Strasbourg, France 